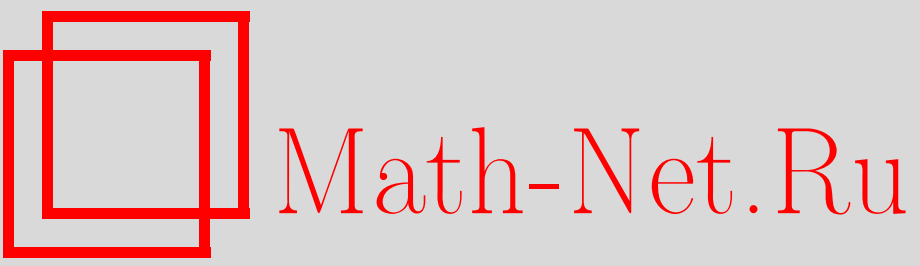

С. Ю. Славянов, Рекуррентные вычисления мультипольных матричных элементов, ТМФ, 1999, том 120, номер 3, 473-481

DOI: https://doi.org/10.4213/tmf791

Использование Общероссийского математического портала Math-Net.Ru подразумевает, что вы прочитали и согласны с пользовательским соглашением

http://www . mathnet.ru/rus/agreement

Параметры загрузки:

IP : 54.80 .97 .219

26 апреля 2023 г., 07:45:09 
ТЕОРЕТИЧЕСКАЯ

И МАТЕМАТИЧЕСКАЯ

ФИЗИКА

Том 120, № 3

сентябрь, 1999

(C) 1999 г.

С. Ю. Славянов*

\section{РЕКУРРЕНТНЫЕ ВЫЧИСЛЕНИЯ МУЛЬТИПОЛЬНЫХ МАТРИЧНЫХ ЭЛЕМЕНТОВ}

Предложен новый метод вычисления степенных (мультипольных) матричных элементов на волновых собственных функциях одномерного уравнения Шредингера. Метод основан на переходе к вспомогательным уравнениям третьего и четвертого порядков и применении к ним аналога преобразования Лапласа. Получившаяся в результате рекуррентная процедура позволяет вычислять матричные элементы через считающиеся известными собственные значения и несколько базовых матричных элементов. В качестве примера рассмотрены мультипольные матричные элементы на волновых функциях гармонического и ангармонического осцилляторов.

Эта работа посвящается памяти создателя иколь математической физики в Санкт-Петербургском государственном университете академика В.А. Фока и семидесятилетию моего учителя, проф. В. С. Булдирева.

\section{ВВЕДЕНИЕ}

В предыдущей публикации автора [1] был предложен метод вычисления диагональных мультипольных матричных элементов на волновых собственных функциях одномерного уравнения Шредингера. Данная публикация расширяет методику на вычисление произвольных недиагональных матричных элементов. В основе метода лежат переход к вспомогательному уравнению четвертого порядка, которому удовлетворяет произведение волновых функций, и применение к нему аналога преобразования Лапласа. Чтобы исключить необходимость обращения к тексту статьи [1], все важнейшие формулы оттуда воспроизводятся в тексте этой статьи. По вопросу же доказательств даны ссылки. Разобранные примеры можно разделить на два типа: а) те, в которых результаты допускают представление в явном виде, и б) те, в которых результаты требуют вычисления (численного или асимптотического) собственных значений, а также некоторых первичных базовых матричных элементов.

Во втором разделе сформулированы и доказаны основные общие леммы и теоремы. В третьем разделе рассмотрены простейшие примеры типа “а", а в четвертом разделе

*Санкт-Петербургский государственный университет, Санкт-Петербург, Россия. E-mail: slav@slav.usr.pu.ru 
- примеры типа "б”. В пятом разделе обсуждаются возможные обобщения предложенной методики на уравнения с весом. Отметим, что история построения рекуррентных соотношений для матричных элементов довольно давняя. Упомянем работу [2], которая была одной из первых игде использовался метод интегрирования по частям, и более современную работу [3], основанную на аппарате теории групп и вычислении коммутаторов.

Пусть $\psi_{n}(z), \lambda_{n}$ - собственные функции и собственные значения задачи на всей оси для одномерного уравнения Шредингера

$$
\begin{gathered}
(-L+\lambda) \psi=\frac{d^{2}}{d z^{2}} \psi(z)+(\lambda-q(z)) \psi(z)=0 \\
\lim \psi(z)_{z \rightarrow-\infty}=0, \quad \lim \psi(z)_{z \rightarrow \infty}=0 .
\end{gathered}
$$

Предполагается выполненным условие

$$
\int_{-\infty}^{\infty} \psi_{n}(z) \psi_{m}(z) d z=\delta_{n m}
$$

где $\delta_{n m}$ - символ Кронекера. Функция $q(z)$ - потенциал в уравнении Шредингера - считается полиномом четного порядка, причем $\left.q(z)\right|_{z \rightarrow \pm \infty} \rightarrow \infty$. Под степенными матричными элементами понимаются интегралы

$$
V_{n m}^{(k)}=\int_{-\infty}^{\infty} z^{k} \psi_{n}(z) \psi_{m}(z) d z
$$

с положительными целыми $k$. В том случае, когда $n=m$, матричные элементы называются диагональными, в противном случае - недиагональными.

\section{2. ОБШАЯ ТЕОРИЯ}

В основе метода лежит получение уравнений, которым удовлетворяют квадрат волновой функции и произведение волновых функций, отвечающих разным собственным значениям. Первым шагом будет доказательство двух простых лемм.

Лемма 1. Квадрат $w_{n n}(z)=\left(\psi_{n}(z)\right)^{2}$ волновой собственной функиии уравнения (1) удовлетворяет уравнению третьего порядка

$$
w_{n n}^{\prime \prime \prime}(z)+4\left(\lambda_{n}-q\right) w_{n n}^{\prime}(z)-2 q^{\prime} w_{n n}(z)=0 .
$$

ДокАЗАТЕЛЬСТВо. Производные функции $w(z)$ имеют вид (индексы для краткости опускаются)

$$
w^{\prime}=2 \psi \psi^{\prime}, \quad w^{\prime \prime}=2\left(\psi \psi^{\prime \prime}+\left(\psi^{\prime}\right)^{2}\right), \quad w^{\prime \prime \prime}=2\left(\psi \psi^{\prime \prime \prime}+3 \psi^{\prime} \psi^{\prime \prime}\right)
$$

Кроме того, из уравнения (1) следует, что

$$
2 \psi^{\prime \prime} \psi^{\prime}=(q-\lambda) w^{\prime}, \quad \psi^{\prime \prime} \psi=(q-\lambda) w, \quad 2 \psi^{\prime \prime \prime} \psi+3(\lambda-q) w^{\prime}=w^{\prime \prime \prime} .
$$


РЕКУРРЕНТНЫЕ ВЫЧИСЛЕНИЯ МУЛЬТИПОЛЬНЫХ МАТРИЧНЫХ ЭЛЕМЕНТОВ 475

Исключив из этих равенств функцию $\psi$, придем к равенству (3).

Рассмотрим два уравнения

$$
y^{\prime \prime}(z)-f(z) y(z)=0, \quad u^{\prime \prime}(z)-g(z) u(z)=0 .
$$

Введем функцию $v(z)$, равную произведению решений этих уравнений, $v=y u$. Согласно этому определению выполнены тождества

$$
\begin{aligned}
v^{\prime} & =y^{\prime} u+y u^{\prime}, \quad v^{\prime \prime}=y^{\prime \prime} u+y u^{\prime \prime}+2 y^{\prime} u^{\prime}, \\
v^{\prime \prime \prime} & =y^{\prime \prime \prime} u+y u^{\prime \prime \prime}+3\left(y^{\prime \prime} u^{\prime}+y^{\prime} u^{\prime \prime}\right), \\
v^{\prime \prime \prime \prime} & =y^{\prime \prime \prime \prime} u+y u^{\prime \prime \prime \prime}+4\left(y^{\prime \prime \prime} u^{\prime}+y^{\prime} u^{\prime \prime \prime}\right)+6 y^{\prime \prime} u^{\prime \prime} .
\end{aligned}
$$

Из уравнений (4) также следует

$$
\begin{gathered}
y^{\prime \prime} u=f v, \quad y u^{\prime \prime}=g v, \quad y^{\prime \prime} u^{\prime \prime}=f g v, \quad 2 y^{\prime} u^{\prime}=v^{\prime \prime}-(f+g) v \\
y^{\prime \prime \prime} u+y^{\prime \prime} u^{\prime}=f^{\prime} v+f v^{\prime}, \quad y u^{\prime \prime \prime}+y^{\prime} u^{\prime \prime}=g^{\prime} v+g v^{\prime} \\
y^{\prime \prime \prime \prime} u+2 y^{\prime \prime \prime} u^{\prime}+y^{\prime \prime} u^{\prime \prime}=f^{\prime \prime} v+2 f^{\prime} v^{\prime}+f v^{\prime \prime} \\
y u^{\prime \prime \prime \prime}+2 y^{\prime} u^{\prime \prime \prime}+y^{\prime \prime} u^{\prime \prime}=g^{\prime \prime} v+2 g^{\prime} v^{\prime}+g v^{\prime \prime}
\end{gathered}
$$

Исключив из этих равенств, насколько возможно, функции $y, u$, получим

$$
\begin{aligned}
& v^{\prime \prime \prime \prime}(z)-2 y^{\prime \prime \prime} u^{\prime}-2 y^{\prime} u^{\prime \prime \prime}-(f+g) v^{\prime \prime}(z)- \\
& \quad-2\left(f^{\prime}+g^{\prime}\right) v^{\prime}(z)-4 f g v(z)-\left(f^{\prime \prime}+g^{\prime \prime}\right) v(z)=0 .
\end{aligned}
$$

Воспользуемся равенствами

$$
y^{\prime \prime} u^{\prime}=f y u^{\prime}, \quad y^{\prime} u^{\prime \prime}=g y^{\prime} u, \quad y^{\prime \prime \prime} u^{\prime}=f y^{\prime} u^{\prime}+f^{\prime} y u^{\prime}, \quad y^{\prime} u^{\prime \prime \prime}=g y^{\prime} u^{\prime}+g^{\prime} y^{\prime} u \text {. }
$$

Положим $f=q-\lambda_{n}, g=q-\lambda_{m}$ и используем равенства $f^{\prime}=g^{\prime}, f^{\prime \prime}=g^{\prime \prime}$. В результате приходим к следуюшему утверждению.

ЛЕмма 2. Произведение $v_{n m}(z)=\psi_{n}(z) \psi_{m}(z)$ волновых собственных функиий (1) удовлетворяет уравнению четвертого порядка

$$
v_{n m}^{\prime \prime \prime \prime}(z)+2\left(\lambda_{n}+\lambda_{m}-2 q\right) v_{n m}^{\prime \prime}(z)-6 q^{\prime} v_{n}^{\prime}(z)+\left(\left(\lambda_{n}-\lambda_{m}\right)^{2}-2 q^{\prime \prime}\right) v_{n m}(z)=0 .
$$

Заметим, что, продифференцировав уравнение (3), получим уравнение (6) при $n=m$. Уравнения (3), (6) могут быть записаны также в виде

$$
T_{4}(z, D) v_{n m}(z)=0, \quad T_{3}(z, D) w_{n n}(z)=0, \quad D \equiv \frac{d}{d z}
$$


где $T_{m}(z, D)$ - полином по обеим переменным $z, D$, причем по переменной $D$ - третьего или четвертого порядка. На функциях $w_{n n}(z), v_{n m}(z)$ определен аналог преобразования Лапласа $\left(w_{n n}(z), v_{n m}(z) \mapsto u_{n m}(p)\right)$ :

$$
\begin{aligned}
& u_{n m}(p)=\int_{-\infty}^{\infty} \exp (-p z) u_{n m}(z) d z \\
& u_{n n}(p)=\int_{-\infty}^{\infty} \exp (-p z) u_{n n}(z) d z
\end{aligned}
$$

Сходимость интегралов гарантируется поведением функций $\psi_{n}(z)$ на бесконечности. Кроме того, согласно свойствам преобразования Лапласа, легко переносимым на используемую модификацию, функция $u_{n m}(p)$ должна удовлетворять уравнению

$$
T\left(D_{p},-p\right) u_{n m}(p)=0
$$

Уравнение (9) имеет в точке $p=0$ регулярную особую точку, однако нужное решение особенности в этой точке не имеет. Противоречия здесь нет, что будет видно из рассмотренных примеров.

Члены разложения функции $u_{n m}(p)$ и есть изучаемые матричные элементы:

$$
u_{n m}(p)=\sum_{0}^{\infty} \frac{1}{k !} V_{n m}^{(k)}(-p)^{k} \equiv \sum_{0}^{\infty} g_{k} p^{k}
$$

Основной результат проведенных выше выкладок можно сформулировать следуюшим образом.

ТЕОремА. Матричные әлементы от волновых собственных функиий $V_{n n}^{(k)}$, отвечающих задаче Штурма-Лиувилля для одномерного уравнения Шредингера (1), с точностью до сомножителей $(-1)^{k} k$ ! совпадают с коэффициентами разложения в ряд Тейлора регулярного в нуле решения уравнения (9).

Доказательство приведено выше.

Сформулированная обшая теорема оставляет большое число вопросов. Действительно, функция $u_{n m}(p)$ регулярна в нуле, но она может иметь в этой точке нуль высокого порядка, что отразится на матричных элементах. Другая конкретная проблема каковы начальные условия для решения рекуррентной системы? Ответы на эти вопросы можно получить, анализируя случай "общего положения" и конкретные примеры со специально выбранным потенциалом $q(z)$. Рассмотрим в этом разделе случай “обшего положения".

ЛЕмма 3. Уравнение (9) для $n \neq m$ имеет вид

$$
\left(p^{2} q\left(D_{p}\right)+\frac{1}{2} p q^{\prime}\left(D_{p}\right)-\frac{1}{4}\left((\delta \lambda)^{2}+2 \Sigma \lambda p^{2}+p^{4}\right)\right) u_{n m}(p)=0
$$


Для доказательства надо написать на основании уравнения (6) уравнение (9) и воспользоваться равенствами

$$
\begin{aligned}
q\left(D_{p}\right) p^{2} u & =p^{2} q\left(D_{p}\right) u+2 p q^{\prime}\left(D_{p}\right) u+q^{\prime \prime}\left(D_{p}\right) u, \\
q^{\prime}\left(D_{p}\right) p u & =p q^{\prime}\left(D_{p}\right) u+q^{\prime \prime}\left(D_{p}\right) u .
\end{aligned}
$$

Уравнение (11) имеет $2 N$-й порядок, совпадаюший с порядком полинома $q(z)$. Точка $p=0$ является регулярной особой точкой уравнения (11) и на формальном уровне имеет характеристическими показателями $\rho=0,1 \ldots, 2 N-1$. Однако из решений нас в общем случае интересует лишь то, которое голоморфно в начале координат и разложение которого в ряд Тейлора начинается с первой степени $p$, т.е. решение

$$
u_{n m}(p)=\sum_{0}^{\infty} g_{k} p^{k+1}
$$

Пусть функция $q^{\prime}(D)$ представляется в виде

$$
q^{\prime}(D)=\sum_{0}^{2 N-1} a_{j} D^{j}
$$

Тогда исходное уравнение, с которого начинается рекуррентная процедура отыскания коэффициентов,

$$
\sum_{1}^{2 N-1} a_{j} j ! g_{j-1}-\frac{1}{4}(\delta \lambda)^{2} g_{0}=0
$$

связывает коэффициенты $g_{0}, \ldots, g_{2 N-2}$. Таким образом, при фиксированных $m, n$, зная численно или асимптотически собственные значения $\lambda_{n}, \lambda_{m}$ и несколько "базовых" матричных элементов (в нашем случае “общего положения" это $g_{0}, \ldots, g_{2 N-3}$ ), остальные мы можем вычислять по рекуррентной процедуре "от начала". Возможно также, полагая, что коэффициенты $g_{k}$ быстро убывают с номером $k$, производить вычисления "от конца". В этом случае нужны только собственные значения.

Аналогом уравнения (11) при $n=m$ является уравнение

$$
\left(p q\left(D_{p}\right)+\frac{1}{2} q^{\prime}\left(D_{p}\right)-\lambda p+\frac{p^{3}}{4}\right) u_{n n}(p)=0
$$

Здесь начальная степень разложения функции $u_{n n}(p)$ в ряд по степеням $p$ всегда фиксирована, поскольку первый матричный элемент - это нормировка функции

$$
u_{n n}(p)=\sum_{0}^{\infty} g_{k} p^{k}
$$

Исследование рекуррентной системы для коэффициентов $g_{k}$ производится так же, как и в случае (11). 


\section{3. ГАРМОНИЧЕСКИЙ ОСЦИЛЛЯТОР}

Рассматривается задача о гармоническом осцилляторе на интервале $(-\infty, \infty)$ :

$$
\psi^{\prime \prime}(z)+\left(\lambda-z^{2}\right) \psi(z)=0, \quad|\psi( \pm \infty)|<\infty
$$

Волновые функции $\psi_{n}$ и собственные значения $\lambda_{n}$ определены соотношениями

$$
\psi_{n}(z)=e^{-z^{2} / 2} H_{n}(z), \quad \lambda_{n}=2 n+1
$$

Функции $H_{n}(z)$ - полиномы Эрмита. Уравнение третьего порядка для квадрата собственной функции имеет вид

$$
w_{n n}^{\prime \prime \prime}(z)+4\left(\lambda-z^{2}\right) w_{n n}^{\prime}(z)-4 z w_{n n}(z)=0
$$

а уравнение четвертого порядка для произведений собственных функций -

$$
v_{n m}^{\prime \prime \prime \prime}(z)+2\left(\lambda_{n}+\lambda_{m}-2 z^{2}\right) v_{n m}^{\prime \prime}(z)-12 z v_{n m}^{\prime}(z)+\left(\left(\lambda_{n}-\lambda_{m}\right)^{2}-4\right) v_{n m}(z)=0
$$

Решение в виде ряда уравнения (18) обсуждается в публикации [1]. Здесь мы рассмотрим решение уравнения (19). Пусть $n-m=l$. Перейдем к уравнению для функции $u_{n m}$ :

$$
p^{2} u_{n m}^{\prime \prime}(p)+p u_{n m}^{\prime}(p)-l^{2} u_{n m}(p)-\left((n+m+1) p^{2}+\frac{p^{4}}{4}\right) u_{n m}(p)=0
$$

Это уравнение второго порядка с регулярной особой точкой в нуле и иррегулярной особой точкой на бесконечности, являющееся частным случаем биконфлюэнтного уравнения Гойна $[4,5]$. Характеристические показатели в нуле равны $\pm l$. Регулярное в нуле решение строится в виде

$$
u_{n m}(p)=\sum_{k=0}^{\infty} c_{k}^{(n m)} p^{l+k}
$$

Подставив решение (21) в уравнение (20), получим трехчленную рекуррентную систему для коэффищиентов $c_{k}$

$$
\begin{aligned}
4(l+1) c_{2}-(n+m+1) c_{0} & =0, \\
k(k+2 l) c_{k}-(n+m+1) c_{k-2}-\frac{1}{4} c_{k-4} & =0 .
\end{aligned}
$$

Из рекуррентной системы (22) видно, что все матричные элементы при фиксированных $n, m$ выражаются через первый отличный от нуля матричный элемент. 


\section{4. АНГАРМОНИЧЕСКИЙ ОСЦИЛЛЯТОР}

Рассмотрим одну из возможных моделей ангармонического осциллятора на $(-\infty, \infty)$ :

$$
\psi^{\prime \prime}(z)+\left(\lambda-t^{2}\left(z^{2}+1\right)^{2}\right) \psi(z)=0, \quad|\psi( \pm \infty)|<\infty .
$$

Уравнение (23) представляет собой частный случай так называемого триконфилюэнтного уравнения Гойна $[4,5]$. Масштабным преобразованием независимой переменной $z \mapsto z t^{-1 / 2}$ оно приводится к более привычной для физиков форме:

$$
\psi^{\prime \prime}(z)+\left(\tilde{\lambda}-\left(2 z^{2}+t^{-1} z^{4}\right)\right) \psi(z)=0,
$$

где $\tilde{\lambda}=\lambda t^{-1}-t$. Введем, как и выше, число $l=n-m$. Тогда четность подынтегральной функции в определении матричного элемента совпадает с $k+l$, и если $k+l$ - нечетное число, то соответствующий матричный элемент равен нулю. Уравнение (8) в рассматриваемом случае имеет вид

$$
\begin{aligned}
& v_{n m}^{\prime \prime \prime \prime}(z)+2\left(\Sigma \lambda-2 t^{2}\left(z^{2}+1\right)^{2}\right) v_{n m}^{\prime \prime}(z)-24 t^{2} z\left(z^{2}+1\right) v_{n m}^{\prime}(z)+ \\
& \quad+\left((\delta \lambda)^{2}-24 t^{2} z^{2}-8 t^{2}\right) v_{n m}(z)=0,
\end{aligned}
$$

где введены обозначения

$$
\Sigma \lambda=\lambda_{n}+\lambda_{m}, \quad \delta \lambda=\lambda_{n}-\lambda_{m} .
$$

После преобразования Лапласа получается уравнение

$$
\begin{aligned}
& p^{2} u_{n m}^{\prime \prime \prime \prime}(p)+2 p u_{n m}^{\prime \prime \prime}(p)+2 p^{2} u_{n m}^{\prime \prime}(p)+2 p u_{n m}^{\prime}(p)+ \\
& \quad+\left(-\frac{(\delta \lambda)^{2}}{4 t^{2}}+\left(1-\frac{\sum \lambda}{2 t^{2}}\right) p^{2}-\frac{p^{4}}{4 t^{2}}\right) u_{n m}(t)=0
\end{aligned}
$$

Точка $z=0$ является регулярной особой точкой уравнения (26). Характеристические числа равны $\rho_{1}=0, \rho_{2,3}=1, \rho_{4}=2$. Одно из решений, отвечаюших корню $\rho_{2,3}=1$, и решение, отвечаюшее корню $\rho_{4}=2$, представляют собой регулярные в нуле функции и могут быть построены в виде рядов Тейлора

$$
\begin{aligned}
& u_{n m}(p)=\sum_{0}^{\infty} c_{k+1} p^{k+1}, \\
& u_{n m}(p)=\sum_{0}^{\infty} d_{k+2} p^{k+2} .
\end{aligned}
$$

Подстановка разложения в уравнение приводит к рекуррентным соотношениям для коэффициентов $c_{k}$

$$
\begin{gathered}
12 c_{3}+\left(-2-\frac{(\delta \lambda)^{2}}{4 t^{2}}\right) c_{1}=0, \\
240 c_{5}+\left(6-\frac{(\delta \lambda)^{2}}{4 t^{2}}\right) c_{3}+\left(1-\frac{\Sigma \lambda}{2 t^{2}}\right) c_{1}=0, \\
(k+6)^{2}(k+7)(k+5) c_{k+7}+\left(2(k+5)(k+3)-\frac{(\delta \lambda)^{2}}{4 t^{2}}\right) c_{k+5}+ \\
+\left(1-\frac{\Sigma \lambda}{2 t^{2}}\right) c_{k+3}-\frac{1}{4 t^{2}} c_{k+1}=0, \quad k=2 j,
\end{gathered}
$$


и для коэффициентов $d_{k}$

$$
\begin{gathered}
72 d_{4}-\left(\frac{(\delta \lambda)^{2}}{4 t^{2}}\right) d_{2}=0 \\
600 d_{6}+\left(16-\frac{(\delta \lambda)^{2}}{4 t^{2}}\right) d_{4}+\left(1-\frac{\Sigma \lambda}{2 t^{2}}\right) d_{2}=0 \\
(k+7)^{2}(k+8)(k+6) d_{k+8}+\left(2(k+6)(k+4)-\frac{(\delta \lambda)^{2}}{4 t^{2}}\right) d_{k+6}+ \\
+\left(1-\frac{\Sigma \lambda}{2 t^{2}}\right) d_{k+4}-\frac{1}{4 t^{2}} d_{k+2}=0, \quad k=2 j .
\end{gathered}
$$

При $n=m$, т.е. в случае диагональных матричных элементов, регулярное в нуле решение допускает представление

$$
u_{n n}(p)=\sum_{0}^{\infty} g_{k} p^{k}
$$

ведущее к следующей рекуррентной системе для коэффициентов:

$$
\begin{gathered}
24 g_{4}+6 g_{2}+\left(1-\frac{\lambda_{n}}{t^{2}}\right) g_{0}=0, \\
(k+4)(k+3)(k+2)^{2} g_{k+4}+(k+2)(2 k+3) g_{k+2}+ \\
+\left(1-\frac{\lambda_{n}}{t^{2}}\right) g_{k}-\frac{1}{4 t^{2}} g_{k-2}=0 .
\end{gathered}
$$

Все четные матричные элементы выражаются через нормировку, и еще один матричный элемент - квадрупольный.

\section{5. ОБОБШЕНИЯ}

Теория и примеры, разобранные в статье, касались одномерного уравнения Шредингера, не содержащего производной. Однако во многих прикладных задачах, возникающих при разделении переменных, мы встречаемся с другой формой уравнения, а именно,

$$
(-L+\lambda) \psi=\frac{d}{d z} r(z) \frac{d}{d z} \psi(z)+(\lambda-q(z)) \psi(z)=0 .
$$

$\mathrm{K}$ таким уравнениям относятся уравнения для классических ортогональных полиномов - полиномов Якоби, Лежандра и Лагерра. Сюда же надо отнести и уравнения, возникающие в более сложных задачах (задача двух кулоновских центров, эффект Штарка на водороде и т.д.). Полное рассмотрение всех этих задач выходит за рамки данной пуб-

ликации. Мы приведем в справочных целях лишь аналог уравнения (3) для квадрата волновой функции

$$
\begin{aligned}
& w_{n n}^{\prime \prime \prime}(z)+3 r^{\prime} w_{n n}^{\prime \prime}(z)+\left(4\left(\lambda_{n}-q\right)+r^{\prime \prime}+\frac{r^{2}}{r}\right) w_{n n}^{\prime}(z)+ \\
& +\left(-2 q^{\prime}+\frac{2(\lambda-q) r^{\prime}}{r}\right) w_{n n}(z)=0
\end{aligned}
$$


и аналог уравнения (6) для произведения волновых функций

$$
\begin{aligned}
& r v_{n m}^{\prime \prime \prime \prime}+5 r^{\prime} v_{n m}^{\prime \prime \prime}+\left(2 \Sigma \lambda-4 q+4 r^{\prime \prime}+\frac{4 r^{\prime 2}}{r}\right) v_{n m}^{\prime \prime}+ \\
& +\left(-6 q^{\prime}-\frac{3(2 q-\Sigma \lambda) r^{\prime}}{r}+r^{\prime \prime \prime}+\frac{3 r^{\prime} r^{\prime \prime}}{r}\right) v_{n m}^{\prime}+ \\
& +\left(\frac{(\delta \lambda)^{2}}{r}-2 q^{\prime \prime}-\frac{4 q^{\prime} r^{\prime}}{r}-\frac{(2 q-\Sigma \lambda) r^{\prime \prime}}{r}\right) v_{n m}=0 .
\end{aligned}
$$

Вывод этих уравнений требует выкладок, аналогичных проведенным при доказательствах лемм 1 и 2, однако более громоздких. Домножив уравнения (34), (35) на $r$, мы приведем их к форме уравнений с полиномиальными коэффициентами. Нетрудно проверить, что дифференцирование уравнения (34) приводит к уравнению, получающемуся из уравнения (35) при $\lambda_{n}=\lambda_{m}$.

Для определения матричных элементов следует, как и выше, перейти к уравнениям, преобразованным по Лапласу, и вычислять разложение решения в нуле.

Благодарности. Работа поддержана Российским фондом фундаментальных исследований, грант №99-01-01362.

\section{Список литературы}

[1] S. Yu. Slavyanov. J. Phys A. 1999. V. 32. P. 1773.

[2] W. Gordon. Ann. Phys. 1929. V. 2. P. 1031.

[3] Н. Ф. Трускова. Линейная алгебра интегралов задачи двух центров в квантовой механике. Препринт Р2-11269. Дубна: ОИЯИ, 1978.

[4] A. Ronveaux. Heun's Differential Equations. Oxford, New York, Tokyo: Oxford University Press, 1995.

[5] А. Зеегер, В. Лай, С. Ю. Славянов. ТМФ. 1995. Т. 104. № 2. С. 233.

Поступила в редакцию 5.V.1999 г.

5 Теоретическая и математическая физика, т. 120, № 3, 1999 г. 